\title{
I'll (Not) be Home for Christmas: The Impact of the Pandemic on Evaluation Apprehension and Self-Disclosure during the 2020 Holidays
}

\author{
Teagen Nabity-Grover \\ Boise State University \\ teagennabitygrov@boisestate.edu
}

\author{
Christy M.K. Cheung \\ Hong Kong Baptist University \\ ccheung@hkbu.edu.hk
}

\author{
Jason Bennett Thatcher \\ Temple University \\ jason.thatcher@temple.edu
}

\begin{abstract}
Social distancing was encouraged and sometimes enforced via lockdowns during the worst of the COVID19 pandemic. However, people still needed to socialize to combat feelings of loneliness, so many turned to social media. While online interactions were encouraged, sharing about behaviors considered unsafe during the pandemic was met with increased shaming and vitriol. This study focuses on understanding whether and why online self-disclosure behaviors changed during the holiday season - a time many people believe should be spent with family and loved ones because of the pandemic. We collected two rounds of survey data in December 2020 from Facebook and Instagram users. Our results show significant differences between the kinds of information disclosed online between 2019 and 2020. We also found that evaluation apprehension moderated the relationship between predicted and reported behaviors for socially desirable information - such as wearing a mask and working from home.
\end{abstract}

Keywords: Social media, Self-disclosure, Pandemic, Holidays, Longitudinal study

\section{Introduction}

The COVID-19 pandemic changed how and what people talked about on social media. For some, social media became a place for users to stay connected with the world - as a source of news and contact with others; this was evidenced by increased usage on Facebook and Instagram (40\%), messaging services - such as WhatsApp and Facebook Messenger (70\%) - Chinese social media apps Weibo and WeChat $(58 \%)$, video services YouTube and TikTok $(\sim 15 \%)$, and social gaming $(31 \%)[1,2,3,4,5]$ from February to April 2020. Nielsen data indicated that social media apps accounted for $25 \%$ of all mobile app usage among US adults beginning in mid-March, up from around $20 \%$ on January 1, 2020 [6]. News outlets attributed the increased time online to combatting depressive episodes and increased levels of anxiety [7, 8, 9].

The rise in social media use was paralleled by a reported rise in social shaming for behaviors deemed socially unacceptable in the context of a pandemic such as dining out and traveling $[10,11,7,8]$. Against a backdrop of changing prescriptions from federal agencies - such as the Center for Disease Control and Prevention (CDC) [12] and the World Health Organization (WHO) [13] - a patchwork of state level actions [14], and conflicting information from government leaders [15], social media users engaged in conversations about (in)appropriate behaviors during the pandemic. In the United States, masks were a particularly divisive topic [16]. We suspect this combination of factors - increased usage of social media and the perceived increase in social shaming - has impacted how individuals use and interact with others on social media.

To understand the interplay of the context of COVID-19 with patterns of social media use, we conducted a longitudinal, quasi-experimental study with Facebook and Instagram users during the 2020 winter holiday season in the United States. The holidays are typically viewed as a time for reconnecting with one's extended social circle [17], so we believed this was a prime time to explore patterns of online self-disclosure. While prior self-disclosure research used a privacy calculus lens to study how users evaluated the personal costs and benefits of revealing personal information online [18, 19], this study considers social impacts on the decision to self-disclose. Using a social calculus lens offers an opportunity for developing a contextualized understanding of self-disclosure during the pandemicafflicted holiday season because of the reported increase in social pressure to adopt and signal preventive behaviors. As such, it affords opportunities to understand how future global health crises impact what and why individuals self-disclose. Our study focuses on how specific kinds of information people disclose online - socially desirable, socially undesirable, and holiday- 
related - has changed and the moderating effect of evaluation apprehension (i.e. how they perceive their social contacts may react to their disclosure) on selfdisclosure intention and behavior. Accordingly, we have two research questions (RQs):

RQ1: How has self-disclosure on social media changed because of the pandemic?

RQ2: How does evaluation apprehension affect the influence of self-disclosure intention on behavior?

Answering these RQs provides insight into how the broader offline and online environments shape social media users' decisions to share information. This helps identify how COVID-19 has affected social media use and informs managers' understanding of possible online user behaviors during future emergencies and health crises. Such insight is important because it affects the reliability of social media data used to inform marketing and advertising decisions. Although this data is specific to the COVID-19 pandemic, the results are likely to be useful for predicting behavior during future pandemics spurred by our increasingly global community and, consequently, integrated biological portmanteau.

\section{Literature Review}

\subsection{Online Self-Disclosure}

Online self-disclosure is defined as any message about the self communicated to another via an online medium [20]; this behavior is considered crucial to relationship development and maintenance as it is how an individual enables another person to learn about them [21]. Although we struggled to find studies concerning self-disclosure during a pandemic, we found studies that were relevant given what news outlets have reported about individuals and their motivations for using social media during this historic time. Specifically, prior studies have found that feelings of loneliness [22, 23], the frequency and duration of social media use [24, 25], and relationship maintenance motivations [26, 27] are positively related to self-disclosure. This means individuals are more likely to share personal information online when they 1) feel lonely, 2) use the social media platform often and for extended periods of time, and 3) want to nurture and/or deepen relationships. Conversely, individuals are less likely to share personal information online if they suspect unwanted audiences may access their content [28].

Furthermore, a recent study argued that the pandemic made individuals more aware of how others evaluate what they disclosed on social media. This phenomenon - referred to as inside-out (i.e. topics not previously common to disclose are now socially encouraged) and outside-in (i.e. topics previously common in disclosures are now discouraged) - indicates that individuals consider more than personal benefits and costs in self-disclosure decisions; they are likely to apply an other-focus (or social calculus lens) in determining what information to share and when [2].

Our literature search uncovered very few studies related to self-disclosure around the holidays. While extant literature indicates holidays are a time for reconnecting with an extended social circle [17], it is unknown how much of that is done online. A study on holiday e-cards revealed it was only deemed acceptable to send an e-card instead of a physical card for far-flung relations and friends [29], though it is unclear how the pandemic may have affected those perceptions. Another study investigating Twitter activity around the Christmas holiday found that Christmas-related tweets numbered nearly one million per day by December 8 , with steady increases beginning around December 14 through a peak on Christmas Eve (December 24); the tweets decreased significantly after December 26 [30]. This behavior seems to echo that observed in an earlier study about social media use before, during, and after a vacation. Many individuals used social media to search for activities and seek out recommendations from their networks before the vacation; about half of the respondents used social media to stay connected with friends during the vacation and $80 \%$ posted about their experiences immediately following their return [31].

\subsection{Evaluation Apprehension}

Evaluation apprehension refers to individuals' concerns about how others evaluate them and their actions $[32,33]$. The fear of retaliation or judgement by others results in people acting in a different manner than they would in normal life. The concept of evaluation apprehension has been used by IS researchers in studying electronic brainstorming systems [33] and group support systems [34].

With the connectivity and transparency of the social media environment [35], evaluation apprehension influences how individuals behave and share information on social media platforms. An individual's actions are easily observed and can be revisited through their profile by others. People care about social rewards and punishment; thus, they are more likely to consider others' perspectives in evaluating the costs and benefits of sharing information [36].

\section{Research Model}

By adopting the social calculus lens [2], we contextualize the exploration of online self-disclosure during the pandemic by identifying three high-level groups of information disclosed during the winter 
holiday season: socially desirable, socially undesirable, and holiday-related. The first group are behaviors that comply with CDC and WHO recommendations for preventing the contraction and spread of COVID-19; as such, we expect this information to be relatively specific to conversations and posts in 2020. The second group of disclosures represent information that was likely to be openly shared before the pandemic but could be perceived as selfish or irresponsible in the midst of the pandemic. Lastly, the holiday-related disclosures are specific to the time of data collection; because holidays are a time for reconnecting and the pandemic conditions discouraged get-togethers and travel, we anticipate these disclosures to be lower in 2020.

In addition to these differences in the kinds and amounts of information shared during the holiday season, we also test the model presented in Figure 1. The relationship between intention and behavior is previously explored in the literature [37, 38, 39], so we anticipate replicating those findings. In other words, intention to disclose will positively influence disclosive behavior.

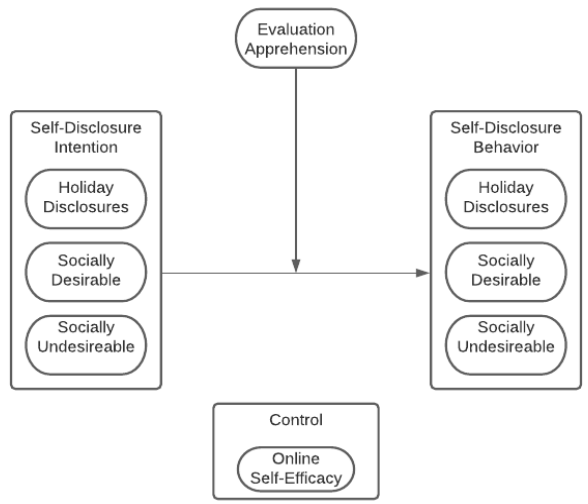

Figure 1. Research model

In considering the social media environment during the pandemic, we argue that it is important to consider how other users influence an individual's decision to disclose information on social media platforms (social calculus lens). Prior research has found that negative evaluation by others in one's social network reduces one's intention to self-disclose [36]. We propose this fear of negative evaluation by others will actually alter the intention-behavior relationship because the nature of social media enables individuals to decide against sharing a disclosure at the last moment - a person may intend to create a new post and decide against doing so with no repercussions [40, 41]. Many social media users will have established patterns of behavior from before the pandemic, so including evaluation apprehension as a moderator allows us to explore disruptions to these established behaviors. Given the reports of increased social shaming in online environments, it's likely evaluation apprehension is more salient than in nonpandemic times. Due to the varying nature of the three groups of disclosures, we suspect evaluation apprehension will operate differently for each, though the literature provides limited guidance in formulating specific hypotheses.

Lastly, we include online self-efficacy as a control variable. Prior studies have found a positive relationship between self-efficacy and self-disclosure - meaning that individuals who feel more capable in using social media and its privacy settings are more likely to disclose personal information on the platform [42, 43]. Given our focus on social influences on the intention-behavior relationship, including self-efficacy as a control is important to limit alternative explanations for the results from model testing - such as competent use of privacy settings.

\section{Method}

Data for this study was collected at two different times using Prolific (https://www.prolific.co/) to recruit and compensate respondents. The first round of data collection occurred on December 19 and 20, 2020 and the survey included items for reported disclosures during the 2019 holiday season, intended disclosures during the 2020 holiday season, online self-efficacy, and evaluation apprehension. Preceding the items concerning 2019 disclosive behaviors, participants were presented with a short primer paragraph reminding them of "business as usual" before the pandemic began. Similarly, before the items concerning 2020 disclosive behaviors, we presented a short primer paragraph describing the current reality during the pandemic, including discussing furloughs, homeschooling, and cancelled travel plans. We followed up with a second survey from December 28, 2020 to January 1, 2021 to measure reported disclosures over the holidays; Prolific's platform enabled us to limit respondents of the second survey to those who completed the first survey. Individuals who completed both surveys were compensated $£ 3.75$ - approximately 5USD.

Our dataset only contains records for respondents who completed both surveys. We removed records that exhibited longstring (consecutively repeated) responses of 19 or more, multiple failed attention checks, and response rates less than two seconds per item. This resulted in a final dataset of 497 usable responses. The following subsections provide additional details on the measures used and the participants of the study. The full instrument is provided in the Appendix. 


\subsection{Measures}

Online self-efficacy was measured using a slightly modified version of the instruments from Compeau and Higgins [44] and Venkatesh and Davis [45] on a sevenpoint sliding Likert scale. Given our social media context, we determined four of the original eight items were well suited to reflecting online self-efficacy in this study with minimal modification. The other four items were greatly modified to reflect confidence in one's ability to use Facebook or Instagram for interacting with others and protecting information shared. Following a pilot test on MTurk, three of the original items and one of the heavily modified items were retained for the full study. The composite reliability for the measure in the full study was .797.

Evaluation apprehension was measured using the three highest loading items from the instrument by La Greca and Lopez [46] on a seven-point sliding Likert scale. We slightly modified two of the items and changed the third item to focus on support instead of liking. No further modifications were made to this measure following the pilot test. The composite reliability for the measure was .940 .

The online self-disclosure measure was developed for this study. While the construct is generally considered to be multi-dimensional - capturing elements of the message (e.g. intimacy, honesty) and behavior (i.e. the intentionality with which information is shared) [20] - we were more concerned with capturing the kind of information being shared rather than measuring more general sharing behaviors. As such, we followed examples from the literature $[47,48]$ and developed a list of nine behaviors that might be shared on social media representing a range of risk given the ongoing pandemic. We included three items we classified as "new and good" behaviors based on CDC recommendations [49], and two items each for low-, moderate-, and high-risk categories based on information published by the British Heart Foundation [50]. Risk, here, is determined by the likelihood one might contract or spread COVID-19 from participating in the specified activity.

These measures were run in a separate pilot study to determine if the classification was valid for further testing. In this study, MTurk respondents were asked to rate on a 10-point scale their perception of being judged or punished for posting the information on social media. This test revealed no statistical difference between moderate- and high-risk behaviors and "new and good" and low-risk behaviors when evaluated for perceived judging and punishment; these two groups of items were statistically different from each other, with the former resulting in higher perceptions of judging and punishment.
Based on these results, we reclassified the moderate- and high-risk items as socially undesirable disclosures; the low-risk and "new and good" items were reclassified as socially desirable disclosures. We developed three additional items that focused on holiday-specific behaviors in which one may partake during the winter season. For each item, respondents indicated if they shared or intended to share the information online (yes/no) and then how frequently they shared (would share) that information on a sixpoint semantic scale from never to every day. We combined these measures into a single value for each item using the following formula: shared * (frequency 1). Subtracting one from the frequency set the "never" response equal to zero from the default value of one, and multiplying by the yes/no response corrects for any misaligned answers between the two responses (e.g. a respondent saying they wouldn't share this information and indicating they shared it once a week). Final values for the disclosure items ranged from zero to five. The composite reliabilities for these measures in the first survey (intended behavior) were .626, .787, and .630 respectively; in the second survey (reported behavior), the composite reliabilities were $.645, .758$, and .529 respectively. While the socially undesirable and holiday behaviors are lower than most recommended cutoffs for reliability, these are new measures and all factor loadings were greater than .404 [51], so we determined these levels acceptable enough to continue our analysis at this time.

All measures exhibited acceptable convergent and discriminant validity.

\subsection{Participants}

Our data included 239 Instagram users and 258 Facebook users recruited through the Prolific platform. Of all participants, $54 \%$ identified as female, $43 \%$ as male, and $3 \%$ as nonbinary or genderqueer. Seventy-two percent identified as white, $15 \%$ as Asian, $7 \%$ as black, and $4 \%$ as multiracial; in terms of ethnicity, $7 \%$ identified as Hispanic or Latinx. Most of our sample was employed full-time at the time of the survey (40\%), $18 \%$ were unemployed, $16 \%$ were students, and $16 \%$ were employed part-time.

Because our survey included items specific to winter holidays, we also asked respondents about their religious affiliation and tendency to celebrate a holiday during the 2020 holiday season - we did not specify a holiday so as to be inclusive of the myriad of celebrations. Half of the respondents indicated they were not religious and another $1 \%$ identified as agnostic; thirty-five percent practiced some form of Christianity, 3\% practiced Judaism, 2\% identified as spiritual, $2 \%$ practiced Hinduism, and $1 \%$ practiced 
each Buddhism, Islam, and Paganism. Ninety-three percent of respondents said they typically celebrated a holiday during December, though only 64\% reported planning to celebrate with family and/or friends during December 2020.

\section{Results}

We analyzed the data using ANOVA in SPSS 26 and structural equation modeling with moderation in MPlus 8.6. First, we review the results of the ANOVA analysis. Then we discuss the results of testing the moderated model.

\subsection{ANOVA Results}

Our data collection provided the disclosure behavior items for three time periods: 2019 holiday season, intended 2020 holiday season, and reported 2020 holiday season. As such, we ran a set of repeated measures ANOVA to examine statistical differences across these times for each classification of disclosure. To do so, we averaged the relevant items for socially desirable, socially undesirable, and holiday behaviors at each time period. In all of these analyses, the assumption of sphericity was violated $(\mathrm{p}=.000)$, so we used the Huynh-Feldt correction [52]; this statistic was significant $(\mathrm{p}=.000)$ in each run, indicating there were significant differences between the means. The results are shown in Figure 2.

Beginning with socially undesirable behaviors (the top chart), there is no statistical difference between the intended (SUI) and reported (SUR) behaviors during the 2020 holiday season $(\mathrm{p}=.713)$; however, the reported behaviors from the 2019 holiday season are significantly higher than those for $2020(\mathrm{p}=.000)$. The socially desirable behaviors (middle chart) demonstrated significant differences at all three periods $(p=.000)$. These behaviors are lowest in 2019 - which is to be expected as some of the items discuss wearing masks and working from home; perhaps more surprising is that respondents expected to share more of these behaviors during the 2020 holiday season (SDI) than they actually reported (SDR) sharing. Lastly, the holiday behaviors (bottom chart) showed significant differences between the three time periods. Behaviors in 2019 were significantly higher than in either of the 2020 periods $(\mathrm{p}=.000)$; the intended holiday disclosures (HolI) were also statistically higher than the reported behaviors (HolR; $\mathrm{p}=.013$ ), though the magnitude is to a smaller degree than with socially desirable behaviors. However, this similarly demonstrates that respondents expected to share more about their holiday plans than they reported sharing on social media at the end of the season.
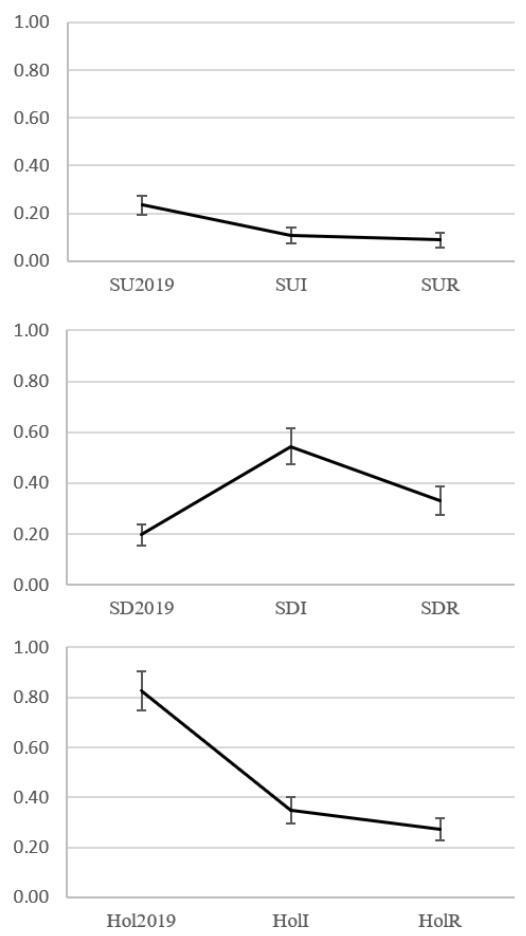

Figure 2. Means and $95 \%$ confidence intervals for information disclosed over time (from top to bottom: socially undesirable, socially desirable, and holidayrelated disclosures)

\subsection{Structural Modeling Results}

Using MPlus software, we ran the structural equation model presented in Figure 1 with and without moderation to compare model fit - the output with moderation only provides AIC and BIC fit statistics; in comparing these statistics, smaller values represent a better model fit. The model with moderation provided a slightly better fit (AIC: 30716.489; BIC: 31154.182) over the model without moderation (AIC: 30737.428; BIC: 31170.912), indicating the moderating effect provided valuable additional information. The results of the moderated model are presented in Figure 3.

It is important to note that the model would not run with a moderator on the relationship between intended and reported socially undesirable disclosures; upon examining the data further, we believe this is because the variance in these two constructs was not significantly different from zero. We tested an alternative calculation based entirely on dummy variables for the socially undesirable items; while this did give us (nonsignificant) results with the moderator, the factor loadings were lower and the analysis generated a warning about the trustworthiness of the standard errors; as such, we do not report a moderation 
estimate for the socially undesirable disclosures, though we have reason to believe it would be nonsignificant.

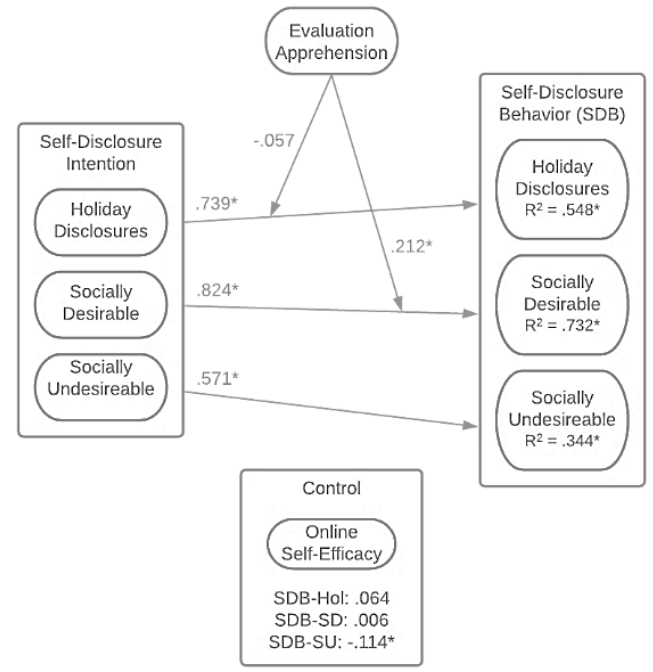

Figure 3. Results of testing the moderated model

As expected, intention was a strong predictor of reported behavior for all three classifications of disclosures. The online self-efficacy control variable was only significant for socially undesirable disclosures. This negative effect indicates individuals who are more aware of how to protect their information online seem to do so by not posting online.

Evaluation apprehension has no significant effect on holiday disclosures. However, it has a significant, positive moderating effect on socially desirable disclosures. This indicates individuals who are more concerned with how others perceive them are likely to share about socially desirable behaviors more often than those who aren't. This interaction is shown in Figure 4. To generate the figure, we calculated the average for each respondent on the three evaluation apprehension items. We then found the overall average (3.18); respondents whose average was higher were assigned to the high group and those whose average was lower were assigned to the low group.

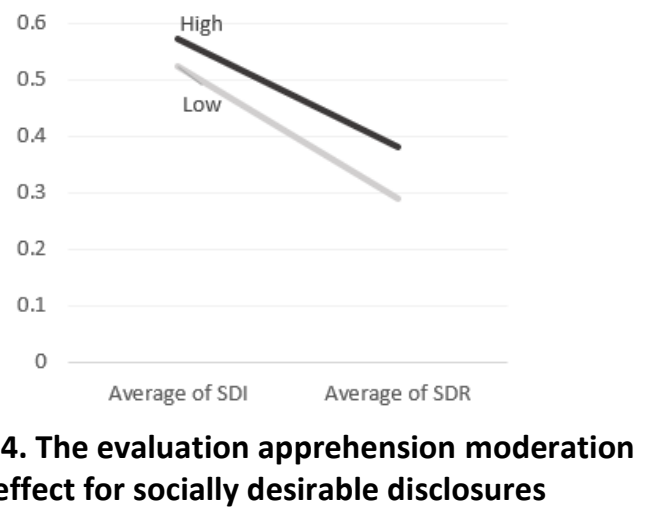

Figure 4. The evaluation apprehension moderation effect for socially desirable disclosures

\section{Discussion}

Against the backdrop of COVID-19 and the 2020 holiday season in the United States, this study explores what and why individuals disclosed different kinds of information on social media. Specifically, we addressed two research questions by identifying different types of information individuals disclose on social media during a pandemic and explaining how evaluation apprehension moderates the effects of self-disclosure intention on behavior.

\subsection{Key Findings}

In a departure from prior work, we suggested that a social calculus lens (i.e. the inside-out and outside-in phenomenon) would drive what and why individuals shared information during the pandemic-afflicted holidays. Perhaps, unsurprisingly, we found the pandemic changed the self-reported holiday season behavior from 2019 to 2020 . By pandemic standards, individuals were more likely to self-disclose socially desirable behaviors in 2020 than in 2019 and less likely to disclose socially undesirable behaviors during the same period. This provides initial support for our contention that a social calculus drives self-disclosure during a pandemic. More importantly, our analysis of evaluation apprehension helps explain why behavior changed during the pandemic. Specifically, we found that evaluation apprehension significantly moderated the relationship between intentions to disclose socially desirable behavior and reported disclosure. We found it did not moderate the relationship between holiday disclosure intention and behavior. While we found a direct relationship from intention to behavior for undesirable information, we were unable to estimate a trustworthy moderating effect.

\subsection{Implications}

These findings on online self-disclosure during COVID-19 have important implications for our understanding of information sharing on social media during pandemics.

First, this study enriches the self-disclosure literature. Specifically, there is some novelty in studying online self-disclosure during the holiday season as this is understudied despite indications that individuals may disclose more during these times. In addition, there is limited understanding in how disclosive behaviors have shifted due to an international health emergency and heightened social pressures [2]; while research conducted before the pandemic suggests we should see increased tendency to disclose personal information 
online $[22,23,24,25,26,27]$, these studies were conducted under a different social climate.

Second, our findings confirm the inside-out and outside-in phenomenon shapes information sharing during a pandemic. Our work explicitly examined intentions to disclose information categorized as holiday-related, socially desirable, and socially undesirable - based on the pandemic context. We found that self-disclosure intention most strongly predicted holiday and socially desirable information sharing. In contrast, our findings imply that, while powerful, intention had less predictive power for disclosure of socially undesirable information sharing behavior. Future work should examine how and why people disclose socially undesirable information which is important because social media is one data source used in contact tracing during outbreaks [53]. Relatedly, future research should also seek to understand which behaviors are considered socially undesirable in various contexts. To increase the usefulness of social media as a data source, we need to understand which activities are unlikely to be discussed and how we might encourage a fuller range of disclosures. This data is also important because firms increasingly rely on social media data to improve shopping and product experiences for customers [54]; if that data is incomplete or key customer segments are underrepresented, the resulting decisions will be untrustworthy.

Third, we found evaluation apprehension offers intriguing insight into how a social calculus shapes information disclosure during a pandemic. Evaluation apprehension did not moderate the relationship for holiday-related disclosure intentions on behavior. This was notable because it suggests that when engaging in social behaviors considered normal or typical of the holidays, the context of COVID-19 did not change this fundamental relationship. However, evaluation apprehension magnified the power of intention on disclosure for socially desirable behaviors. This may indicate that social influences can disrupt previously established patterns in the intention-behavior relationship on social media. This finding is also important because it suggests individuals may disclose socially desirable behaviors to avoid negative judgements and/or reap praise for openly performing and supporting these behaviors. Future work investigating the nuance of these two motivations could be important; whether individuals disclose behavior due to fear of judgement or desire for social capital influences how policy makers and organizations can effectively encourage social media users to respond appropriately to future pandemic situations. For example, such insight could inform whether health officials emphasize fear of what could happen from not complying with health guidelines or the benefits of complying with health guidelines during a pandemic [55].

\section{Limitations and Future Research}

As with all research, our study is not without limitations. First, the study we report here is subject to bias from use of a single method - survey. This especially limits the kinds of activities disclosed during the data collection periods. However, we have tried to balance this by collecting data at a second time period shortly after most December holidays had concluded.

Second, we rely on self-reported behaviors in the survey. We used priming in the initial survey to help distinguish between the 2019 and 2020 holiday seasons before asking participants to respond. This priming included an invitation for the respondent to review their timeline from 2019 to remember what kinds of information they posted, though we have no way to know if participants did so. With the second data collection following after the conclusion of most holidays, it is likely these reports are more trustworthy due to recency bias [56].

Third, our survey does not capture more detailed information on participants' locations within the United States. However, around the holiday season, most cities and states were in various stages of reopening and all were permitting interstate travel. Although we cannot account for exact differences in local government pandemic responses based on location, we feel the situation in the United States was approximately the same across the country at the time of collection.

Fourth, the data collection coincided with a hotly contested election in the United States. Although holidays are often considered times for family (94\% of our sample somewhat to strongly agreed with this statement) and encourages reconnecting with one's network [17], political tensions were quite high and divisive as the election results faced ongoing contestation [57, 58]. Consequently, one could argue our findings are a result of the pandemic and the election. However, because all respondents were residents of the United States, they all faced the same political climate and so all results should be similarly affected by this outside influence.

Our study and these limitations suggest several directions for future research. First, building on the results of our study, it would be beneficial to continue exploring the effects of social calculus (i.e. inside-out and outside-in phenomenon) on self-disclosure behaviors. Privacy calculus is extensively studied in attempting to understand online sharing behaviors, though the results are often mixed and contradictory [20]. However, social calculus may be able to explain aspects of these behaviors that are as yet less understood 
by researchers, including the differences between adolescents and adults. This research may explore social calculus constructs and their impact on online selfdisclosure, but may also continue exploring motivators for socially (un)desirable disclosures.

Second, we face a unique opportunity to continue studying online disclosive behaviors during a global pandemic. As vaccinations are distributed worldwide, it will be interesting to explore how this change impacts disclosure decisions and perceptions of socially (un)desirable behaviors. Additionally, it will be important to determine if the effects of the pandemic are enduring. The pandemic clearly encouraged sharing information that wasn't previously shared as openly or broadly, and discouraged sharing other kinds of information that was more commonplace - as revealed in our ANOVA analyses. As our world recovers from the recent pandemic, how do perceptions of socially (un)desirable disclosures change? Has the past year permanently changed how much individuals are willing to disclose certain kinds of information?

Third, the pandemic reached the United States during a divisive time in its political history. It may be valuable to analyze actual social media posts, paired with interviews, to disentangle the effects of the pandemic from those of the political environment. Given the pandemic was politicized in the United States [16], it may be interesting to compare social media habits in the United States to those in another country less affected by politics in its pandemic response.

\section{Conclusion}

This study explored what and why social media users disclosed during the 2020 holiday season in the midst of a pandemic. We found evidence that a) the kind of information disclosed changed during the 2020 holiday season compared to reported 2019 disclosures and b) social calculus drove decisions to share about socially desirable behaviors. While we found intention explained a moderate amount of variance in disclosing socially undesirable behaviors, our findings suggest a need for developing more nuanced explanations for understanding this information sharing behavior. Additionally, we believe future research should continue exploring online self-disclosure through a social calculus lens and determine which, if any, effects of the pandemic are enduring. Both of these may have major implications for firms and the management of future health crises.

\section{References}

[1] Holmes, R., "Is COVID-19 Social Media's Levelling Up Moment?", Forbes, 2020. https://www.forbes.com/ sites/ryanholmes/2020/04/24/is-covid-19-socialmedias-levelling-up-moment/\# 32e022256c60

[2] Nabity-Grover, T., C.M.K. Cheung, and J.B. Thatcher, "Inside out and outside in: How the COVID-19 pandemic affects self-disclosure on social media", International Journal of Information Management, 2020, pp. 102188.

[3] Perez, S., "Kids now spend nearly as much time watching TikTok as YouTube in US, UK and Spain ", TechCrunch, 2020. https://techcrunch.com/2020/06/ 04/kids-now-spend-nearly-as-much-time-watchingtiktok-as-youtube-in-u-s-u-k-and-spain/

[4] Perez, S., "Report: WhatsApp has seen a $40 \%$ increase in usage due to COVID-19 pandemic | TechCrunch", TechCrunch, 2020. https://techcrunch.com/2020/03/ 26/report-whatsapp-has-seen-a-40-increase-in-usagedue-to-covid-19-pandemic/

[5] Koeze, E., and N. Popper, "The virus changed the way we Internet", The New York Times, 2020. https://www.nytimes.com/interactive/2020/04/07/tech nology/coronavirus-internet-use.html

[6] Fischer, S., "Social media use spikes during pandemic", Axios, 2020. https://www.axios.com/social-mediaoveruse-spikes-in-coronavirus-pandemic-764b384da0ee-4787-bd19-7e7297f6d6ec.html

[7] Harris, M., "Coronavirus has amplified online shaming not just for influencers", Insider, 2020. https://www.insider.com/coronavirus-shaming-viralcallout-online-pandemic-behavior-covid-2020-4

[8] St. Michel, P., "Understanding the need to shame someone on social media for not exercising self-restraint during a pandemic", The Japan Times, 2020. https://www.japantimes.co.jp/news/2020/05/16/nation al/media-national/social-media-shaming-coronavirus/

[9] Snyder, V., "What marketers need to know about people's social media patterns during the pandemic", business.com, 2020. https://www.business.com/articles /social-media-patterns-during-the-pandemic/

[10] Brown, J., "Social media shaming is spiking during the coronavirus pandemic, for better or worse", The Colorado Sun, 2020. https://coloradosun.com/2020/04/ 01/social-media-shaming-about-coronavirus/

[11] Fox, L., "COVID fears spawn social media shaming", The Alpena News, 2020. https://www.thealpenanews. com/news/local-news/2020/04/covid-fears-spawnsocial-media-shaming/

[12] Brooks Pierce, N. Sanders, and J. Thaller-Moran, "The CDC (and Others) Change Guidance", JD Supra, 2020. https://www.jdsupra.com/legalnews/the-cdc-andothers-change-guidance-92421/

[13] World Health Organization, “Timeline: WHO's COVID19 Response", 2021. https://www.who.int/emergencies /diseases/novel-coronavirus-2019/interactivetimeline\#!

[14] Price, A., and L. Myers, Federal, State, and Local Government Responses to COVID-19, 2020.

[15] Kolata, G., and R.C. Rabin, “'Don't be afraid of COVID,' Trump says, Undermining public health messages", The New York Times, 2020. https://www.nytimes. com/2020/10/05/health/trump-covid-publichealth.html 
[16] McKelvey, T., "Coronavirus: Why are Americans so angry about masks?", BBC News, 2020. https://www.bbc.com/news/world-us-canada53477121

[17] Searle-Chatterjee, M., "Christmas cards and the construction of social relations in Britain today", In D. Miller, ed., Unwrapping Christmas. Clarendon Press, Oxford, 1993, 176-192.

[18] Cheung, C., Z.W.Y. Lee, and T.K.H. Chan, "Selfdisclosure in social networking sites: The role of perceived cost, perceived benefits and social influence", Internet Research 25(2), 2015, pp. 279299.

[19] Wang, T., T.D. Duong, and C.C. Chen, "Intention to disclose personal information via mobile applications: A privacy calculus perspective", International Journal of Information Management 36(4), 2016, pp. 531-542.

[20] Nabity-Grover, T.M., "Sharing Is Caring: Essays on Online Self-Disclosure", ProQuest Dissertations and Theses, 2020.

[21] Altman, I., and D.A. Taylor, Social penetration: The development of interpersonal relationships, Holt, Rinehart \& Winston, 1973.

[22] Al-Saggaf, Y., and S. Nielsen, "Self-disclosure on Facebook among female users and its relationship to feelings of loneliness", Computers in Human Behavior 36, 2014, pp. 460-468.

[23] Lee, K.-T., M.-J. Noh, and D.-M. Koo, "Lonely People Are No Longer Lonely on Social Networking Sites: The Mediating Role of Self-Disclosure and Social Support", Cyberpsychology, Behavior, and Social Networking 16(6), 2013, pp. 413-418.

[24] Chang, C.-W., and J. Heo, "Visiting theories that predict college students' self-disclosure on Facebook", Computers in Human Behavior 30, 2014, pp. 79-86.

[25] Leung, L., "Loneliness, Self-Disclosure, and ICQ ('I Seek You') Use”, Cyberpsychology \& Behavior 5(3), 2002, pp. 241-251.

[26] Heravi, A., S. Mubarak, and K.-K.R. Choo, "Information privacy in online social networks: Uses and gratification perspective", Computers in Human Behavior 84, 2018, pp. 441-459.

[27] Hollenbaugh, E.E., and A.L. Ferris, "Facebook selfdisclosure: Examining the role of traits, social cohesion, and motives", Computers in Human Behavior 30, 2014, pp. 50-58.

[28] Zhao, C., P. Hinds, and G. Gao, "How and to whom people share: the role of culture in self-disclosure in online communities", Proceedings of the ACM 2012 Conference on Computer Supported Cooperative Work, (2012), 67-76.

[29] Gooch, D., and R. Kelly, "Season's greetings: An analysis of Christmas card use", Proceedings of the $2016 \mathrm{CHI}$ Conference Extended Abstracts on Human Factors in Computing Systems, Association for Computing Machinery (2016), 2105-2111.

[30] Hu, W., "Real-Time Twitter Sentiment toward Thanksgiving and Christmas Holidays", Social Networking 02(02), 2013, pp. 77-86.

[31] Fotis, J., D. Buhalis, and N. Rossides, "Social Media Impact on Holiday Travel Planning: The Case of the
Russian and the FSU Markets", International Journal of Online Marketing 1(4), 2011, pp. 1-19.

[32] Diehl, M., and W. Stroebe, "Productivity Loss In Brainstorming Groups: Toward the Solution of a Riddle", Journal of Personality and Social Psychology 53(3), 1987, pp. 497-509.

[33] Pinsonneault, A., H. Barki, R.B. Gallupe, and N. Hoppen, "Electronic Brainstorming: The Illusion of Productivity", Information Systems Research 10(2), 1999, pp. 110-133.

[34] Pinsonneault, A., and N. Heppel, "Anonymity in Group Support Systems Research: A New Conceptualization, Measure, and Contingency Framework", Journal of Management Information Systems 14(3), 1997, pp. 89108.

[35] Kane, G.C., M. Alavi, G. (Joe) Labianca, and S.P. Borgatti, "What's Different About Social Media Networks? A Framework and Research Agenda", MIS Quarterly 38(1), 2014, pp. 275-304.

[36] Wagner, A., H. Krasnova, O. Abramova, P. Buxmann, and I. Benbasat, "From 'Privacy Calculus' to 'Social Calculus': Understanding Self-Disclosure on Social Networking Sites", Thirty Ninth International Conference on Information Systems, (2018).

[37] Cheung, C.M.K., and M. Limayem, "The Role of Habit in Information Systems Continuance: Examining the Evolving Relationship Between Intention and Usage", ICIS 2005 Proceedings 39, 2005, pp. 471-482.

[38] Keith, M.J., S.C. Thompson, J. Hale, P.B. Lowry, and C. Greer, "Information disclosure on mobile devices: Reexamining privacy calculus with actual user behavior", International Journal of Human-Computer Studies 71(1163-1173), 2013.

[39] Limayem, M., S.G. Hirt, and C.M.K. Cheung, "How Habit Limits the Predictive Power of Intention: The Case of Information Systems Continuance", MIS Quarterly 31(4), 2007, pp. 705-737.

[40] Ellison, N.B., and J. Vitak, "Social Network Site Affordances and Their Relationship to Social Capital Processes", In S.S. Sundar, ed., The Handbook of the Psychology of Communication Technology. WileyBlackwell, Boston, 2015, 205-227.

[41] Walther, J., "Computer-mediated communication: Impersonal, interpersonal, and hyperpersonal", Communication Research 23, 1996, pp. 1-43.

[42] Chen, H.-T., "Revisiting the Privacy Paradox on Social Media With an Extended Privacy Calculus Model: The Effect of Privacy Concerns, Privacy Self-Efficacy, and Social Capital on Privacy Management", American Behavioral Scientist 62(10), 2018, pp. 1392-1412.

[43] Yu, J., P.J.-H. Hu, and T.-H. Cheng, "Role of Affect in Self-Disclosure on Social Network Websites: A Test of Two Competing Models.", Journal of Management Information Systems 32(2), 2015, pp. 239-277.

[44] Compeau, D.R., and C.A. Higgins, "Computer SelfEfficacy: Development of a Measure and Initial Test.”, MIS Quarterly 19(2), 1995, pp. 189-211.

[45] Venkatesh, V., and F.D. Davis, "A Model of the Antecedents of Perceived Ease of Use: Development and Test", Decision Sciences 27(3), 1996, pp. 451-481.

[46] La Greca, A.M., and N. Lopez, "Social Anxiety among 
Adolescents: Linkages with Peer Relations and Friendships", Journal of Abnormal Child Psychology 26(2), 1998, pp. 83-94.

[47] Attrill, A., and R. Jalil, "Revealing only the superficial me: Exploring categorical self-disclosure online", Computers in Human Behavior 27(5), 2011, pp. 1634 1642.

[48] Ma, X., J. Hancock, and M. Naaman, "Anonymity, intimacy and self-disclosure in social media", Conference on Human Factors in Computing Systems Proceedings, (2016).

[49] Weiner, R., and T. Telford, "CDC issues guidelines that could change the way we work", The Washington Post, 2020. https://www.washingtonpost.com /health/cdcissues-guidelines-that-could-change-the-way-wework/2020/05/29/31fc744a-a1c1-11ea-95901858a893bd59_story.html

[50] British Heart Foundation, "What Everyday Activities Put You at Risk of Getting Coronavirus?", Heart Matters, 2020. https://www.bhf.org.uk/informationsupport/ heart-matters-magazine/news/behind-the-headlines/ coronavirus/everyday-activities-and-the-risk-fromcoronavirus

[51] Thatcher, J.B., R.T. Wright, H. Sun, T.J. Zagenczyk, and R. Klein, "Mindfulness in Information Technology Use: Definitions, Distinctions, and a New Measure", MIS Quarterly 42(3), 2018.

[52] Field, A., Discovering Statistics Using IBM SPSS Statistics, SAGE, London, 2013.

[53] Agbehadji, I.E., B.O. Awuzie, A.B. Ngowi, and R.C. Millham, "Review of big data analytics, artificial intelligence and nature-inspired computing models towards accurate detection of COVID-19 pandemic cases and contact tracing", International Journal of Environmental Research and Public Health 17(15), 2020 , pp. $1-16$.

[54] Ibrahim, N.F., and X. Wang, "Decoding the sentiment dynamics of online retailing customers: Time series analysis of social media", Computers in Human Behavior 96, 2019, pp. 32-45.

[55] Rogers, R.W., "A protection motivation theory of fear appeals and attitude change", The Journal of Psychology 91(1), 1975, pp. 93-114.

[56] Martin, E., Survey Questionnaire Construction, Washington, D.C., 2006.

[57] Fandos, N., and M.S. Schmidt, "Trump Allies Eye LongShot Election Reversal in Congress, Testing Pence", The New York Times, 2020. https://www.nytimes.com /2020/12/13/us/politics/trump-allies-election-overturncongress-pence.html

[58] Mascaro, L., E. Tucker, M.C. Jalonick, and Taylor Andrew, "Pro-Trump mob storms US Capitol in bid to overturn election", Associated Press, 2021. https://apnews.com/article/congress-confirm-joebiden-78104aea082995bbd7412a6e6cd13818

\section{Appendix}

The items included for disclosive behaviors are from the first survey in regards to intended behaviors during the 2020 holiday season; each item started with "This holiday season...." The 2019 items started with "Last holiday season...", the items on the second survey started with "During the recent holiday season...", and both had modified verb tenses.

Online Self-Efficacy (*significantly modified)

1. I could use privacy settings on [Instagram/Facebook] if there was no one around to tell me what to do as I go.

2. I am confident in my ability to protect my information on [Instagram/Facebook].*

3. I could use privacy settings on [Instagram/Facebook] if I had only online guides for reference.

4. I could use privacy settings on [Instagram/Facebook] if I had seen someone else using them before trying it myself.

\section{Evaluation Apprehension}

1. I worry about what others would say about me.

2. I worry that others would not like me.

3. I am afraid that others would stop supporting me. Disclosive Behaviors (^socially undesirable, $\sim$ socially desirable, the rest are holiday items)

1. ...I will share details about going to the beach on [Instagram/Facebook].^

2. ...I will discuss attending a religious service at the place of worship on [Instagram/Facebook].^

3. ...I will share about going to a salon or barber for a haircut on [Instagram/Facebook].^

4. ...I will openly share about working out at the gym on [Instagram/Facebook].^

5. ...I will share about washing my hands frequently on [Instagram/Facebook].

6. ...I will talk about buying groceries at the store on [Instagram/Facebook].

7. ...I will post about ordering delivery from a restaurant on [Instagram/Facebook].

8. ...I will discuss wearing a mask on [Instagram/Facebook].

9. ...I will discuss working from home on [Instagram/Facebook].

10. ...I will post on [Instagram/Facebook] about going out to look at holiday light displays in my town.

11. ...I will openly share on [Instagram/Facebook] about attending local outdoor festivities (e.g. a tree lighting).

12. ...I will share about holiday parties with friends/family on [Instagram/Facebook]. 